\title{
Sliding History-Dependent Adhesion of Nanoscale Silicon Contacts Revealed by in situ Transmission Electron Microscopy
}

\author{
Supplementary Information
}

\section{Statistical values from individual groups of experiments}

Table 1 provides relevant statistical information sampled by group and over the whole set of data.

Table 1: Statistical values from individual groups of experiments and from the data set as a whole

\begin{tabular}{|c|c|c|c|c|c|}
\hline group & $\begin{array}{c}\% \text { of } \mathrm{C}^{-\mathrm{S}^{1}} \\
\text { tests that showed } \\
\text { undetectable adhesion }\end{array}$ & $\begin{array}{l}\% \text { of C-S-S } \mathrm{S}^{2} \text { tests that } \\
\text { had higher adhesion } \\
\text { than the highest } \\
\text { adhesion in C-S tests }\end{array}$ & $\begin{array}{c}\text { Mean of DMT } W_{\text {adh }} \text { for } \\
\text { C-S tests }\left[\mathrm{mJ} / \mathrm{m}^{2}\right]\end{array}$ & $\begin{array}{l}\text { Mean of DMT } \mathrm{W}_{\mathrm{adh}} \text { for } \\
\text { C-S-S tests }\left[\mathrm{mJ} / \mathrm{m}^{2}\right]\end{array}$ & $\begin{array}{l}\text { Average factor of } \\
\text { increase of adhesion } \\
\text { from sliding }\end{array}$ \\
\hline 1 & 65 & 70 & $4.4 \pm 10.8$ & $140.8 \pm 141.7$ & 31.8 \\
\hline 2 & 63 & 43 & $1.2 \pm 2.9$ & $14.8 \pm 10.3$ & 12.7 \\
\hline 3 & 31 & 95.7 & $20.3 \pm 25.6$ & $370.7 \pm 127.1$ & 18.2 \\
\hline 4 & 30 & 64 & $15.2 \pm 19.7$ & $248.5 \pm 177.3$ & 16.3 \\
\hline 5 & 60 & 61 & $1.7 \pm 3.3$ & $53.1 \pm 66.1$ & 31.6 \\
\hline 6 & 72 & 28 & $9.5 \pm 19.3$ & $44.9 \pm 34.0$ & 4.7 \\
\hline Average & 53.5 & 60.3 & 8.7 & 145.5 & 19.2 \\
\hline Standard Deviation & 18.3 & 23.3 & 7.8 & 139.4 & \\
\hline
\end{tabular}

${ }^{1} \mathrm{C}$-S refers to Contact-Separate tests ${ }^{2} \mathrm{C}-\mathrm{S}$-S refers to Contact-Slide-Separate tests

\section{Tip Radius Measurements}

Table 2 lists the tip radius and uncertainty for each group. The tips profiles were traced up to $5 \mathrm{~nm}$, from their apex and then fit with circles. This procedure was repeated for a total of 5 times and the uncertainty is the standard deviation of the radius from this set.

\begin{tabular}{|c|c|c|}
\hline Group & Radius [nm] & Uncertainty [nm] \\
\hline 1 & 310 & 12 \\
\cline { 2 - 3 } 2 & 2,370 & 15 \\
\cline { 2 - 3 } 3 & 154 & 9 \\
\cline { 2 - 3 } 4 & 150 & 15 \\
5 & 886 & 16 \\
\cline { 2 - 3 } 6 & 964 & 22 \\
\cline { 2 - 3 } & &
\end{tabular}




\section{Probe images for each experiment}

Figure S1 shows TEM images of the probes used in the six groups of experiments conducted in this work.

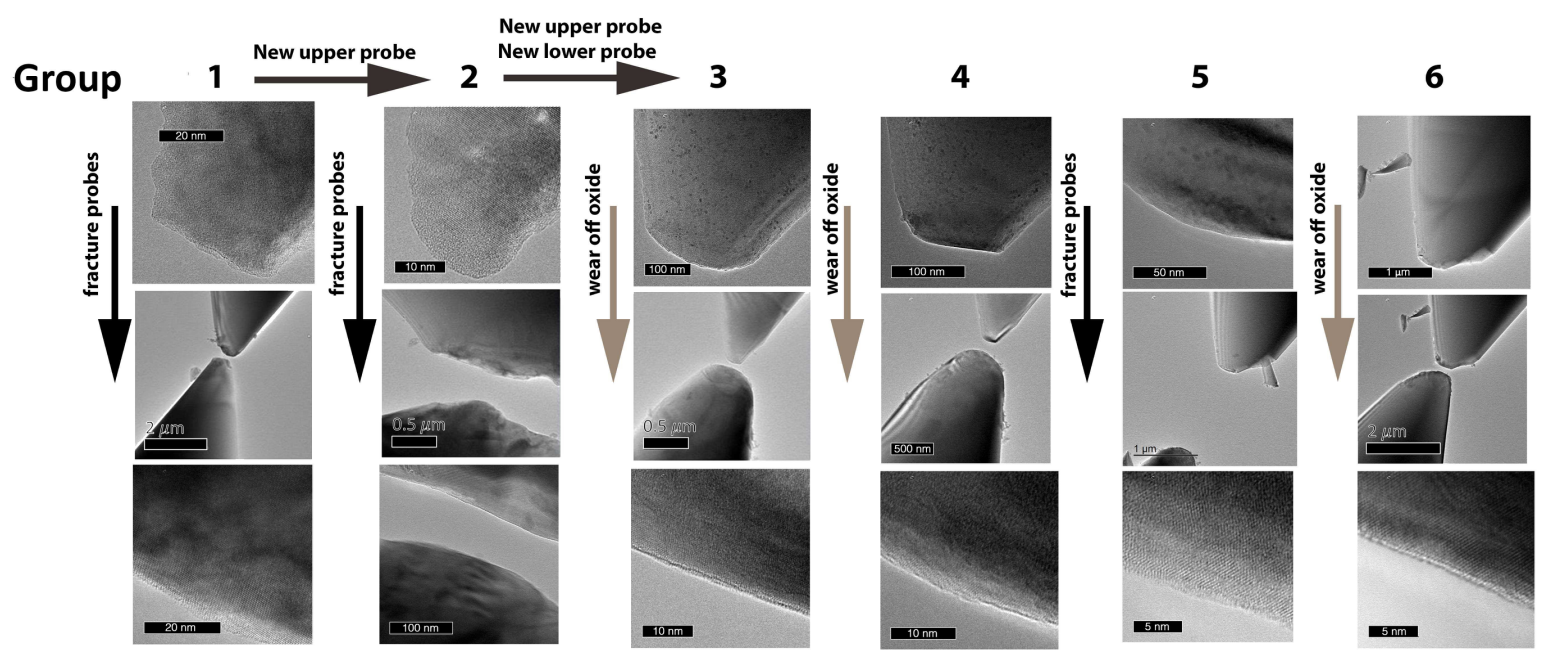

Figure S1: Images of the probes used in the 6 groups of experiments (columns labeled by experiment number) taken before all experiments in that group (first row), during a representative experiment in that group (middle row), and after a representative experiment in that group (bottom row). Down arrows indicate whether the oxide was removed via wear or fracture and right arrows indicate when a different probe from the previous probe was used.

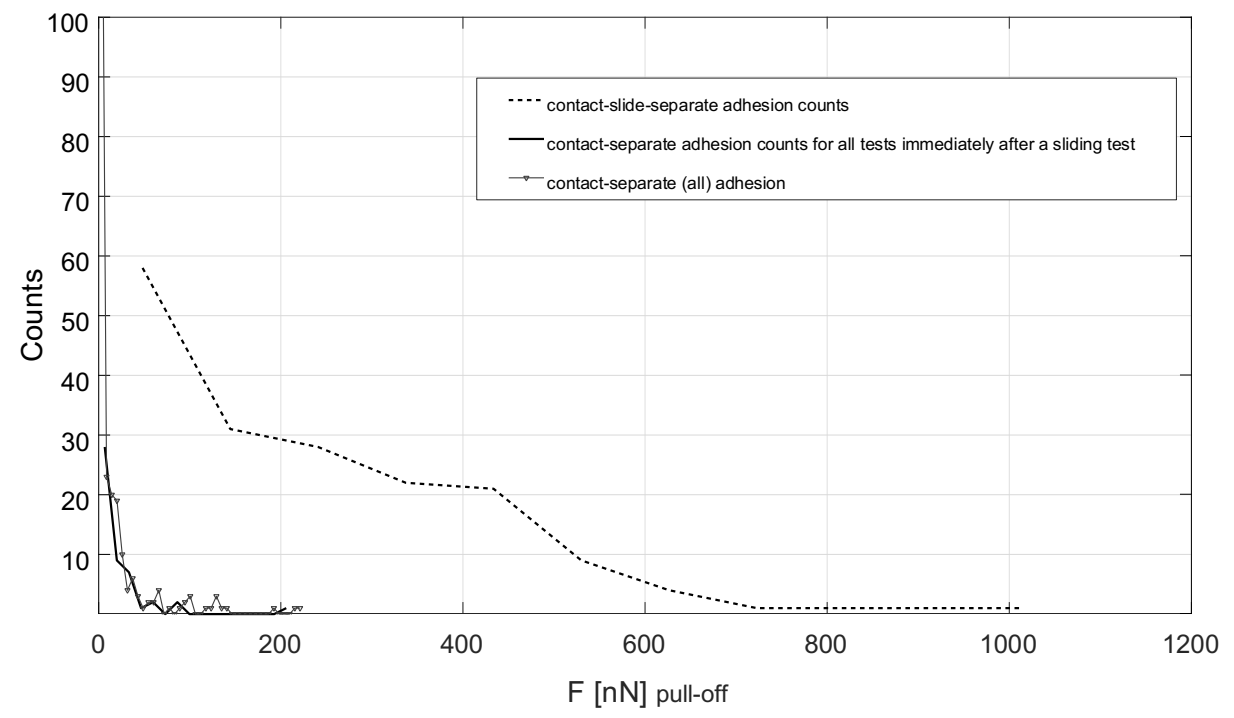

Figure S2: $F_{\text {pull-off }}$ histograms for all contact-slide-separate tests as well as for all contact-separate tests that were the first ones performed after a contact-slide-separate test. The histogram for all contact-separate tests is included, showing that its subset of the contact-separate tests that were the first ones performed after a contact-slideseparate test is statistically similar to it. 


\section{Statistics of the first contact-separate tests immediately after contact-slide-separate test}

Figure $\mathrm{S} 2$ shows adhesion ( $\left.F_{\text {pull-off }}\right)$ histograms for all contact-separate tests that were the first tests done after a contact-slide-separate test. The time elapsed between the contact-slide-separate test and the next contact-separate test ranged from 5 to 60 seconds. Results for contact-slide-separate tests are included for comparison. The low adhesion values for such contact-separate tests indicates that the sliding-induced removal of passivation is shortlived, and therefore generally only observable immediately after sliding.

\section{Discussion of the role of wear particles}

As mentioned in the main text, while most posterior surfaces remained particle-free, after some contactseparate and contact-slide-separate tests, particles 5-7 nm in size were observed on the surfaces of the upper and/or lower tips. Figure S3 shows two instances of pristine upper tip surface, and one of a surface decorated with a few particles, all taken after contact-slide-separate tests. A close inspection of TEM images and videos reveals that these particles had no effect on the adhesive process. The videos clearly show that in all tests there was intimate contact between the two tip surfaces without particle interference; the particles were outside of the contact zone. For three contact-separate tests, particles at the edges of the contact were transferred from one probe to the other. Adhesion coinciding with these cases was less than $5 \mathrm{nN}$, which indicates that the particles did not increase adhesion substantially. Figure $\mathrm{S} 4$ shows one example of this. Since the surfaces 


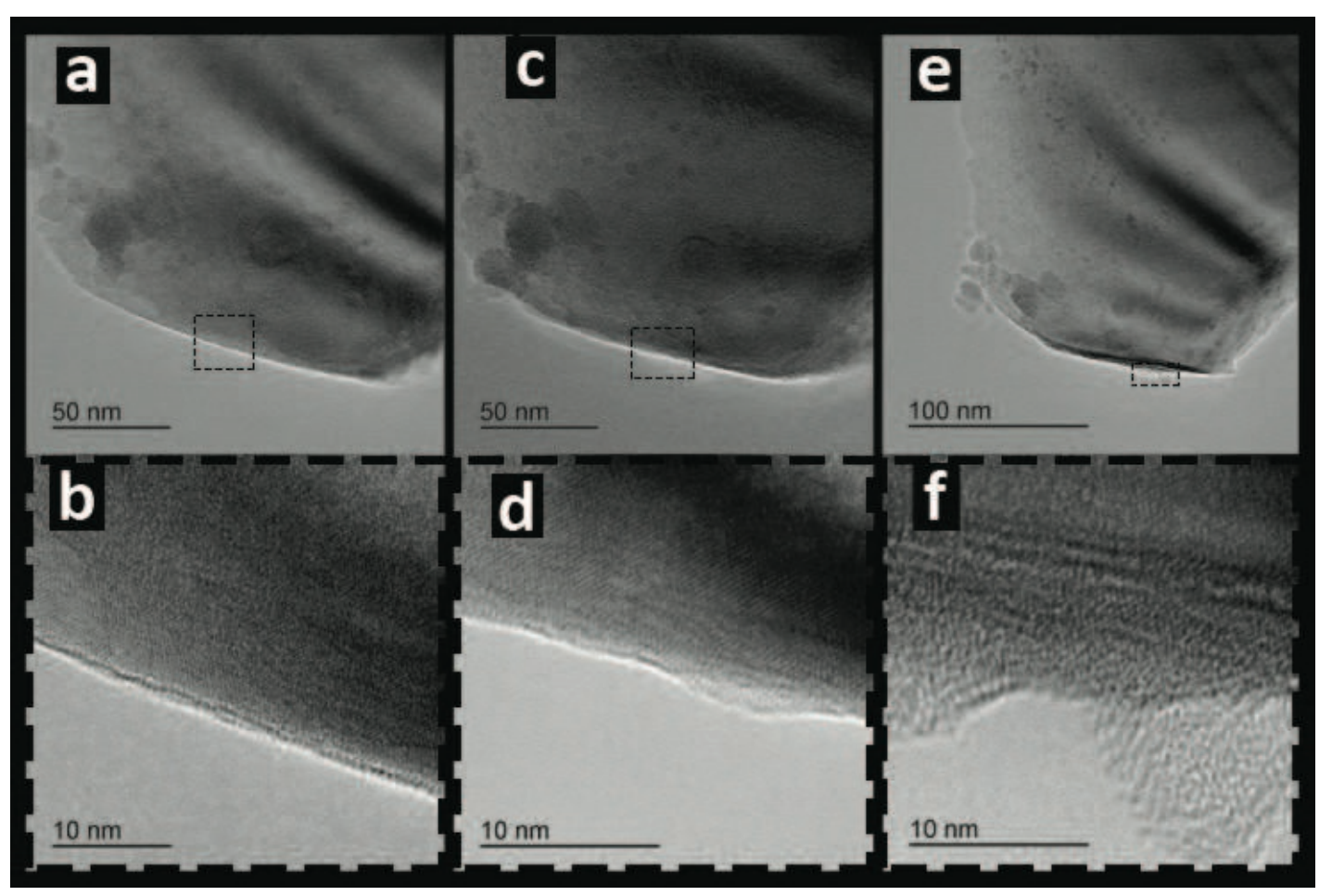

Figure S3: Selected images of the upper probe with and without particles. $\mathbf{a}, \mathbf{b}$,: Low and high magnification images of the upper probe from the third group after a contact-slide-separate test. $\mathbf{c}, \mathbf{d}$,: Low and high magnification images of the upper probe from the third group after another contact-slide-separate test. The apex in these images is also crystalline. e, f: Low and high magnification images the third probe after one contact-slide-separate test showing particles or wear debris on the apex. The large dark particle on the left shank are Si fracture debris. Dashed boxes in low magnification images in the top row indicate the region where the high magnification images of the bottom row were taken.

were initially pristine, the particles were possibly created by large adhesion forces pulling out damaged material from the opposite surface. Thus, particles are symptomatic of adhesive interactions between the tips, rather than causing such adhesion.

Figure S3 (e) and (f) show debris in the contact region, which suggests they were pulled out of the lower surface. The videos show that intimate contact is made during sliding, thus particles must be swept aside so that the contacts were always free of material other than pure silicon.

\section{Pull-off force uncertainty}

Since the pull-off force depends linearly on the pull-off distance i.e., $F_{\text {pull-off }}=k_{n} * d_{\text {pull-off, }}$ the uncertainty in the pull-off force, $\Delta F_{\text {pull-off, is }}$ 


$$
\Delta F_{p u l l-o f f}=\sqrt{\left(k_{n} \Delta d p u l l-o f f\right)^{2}+\left(d p u l l-o f f \Delta k_{n}\right)^{2}} .
$$

The uncertainty in the spring constant from the Sader method is estimated to be ten percent of the spring

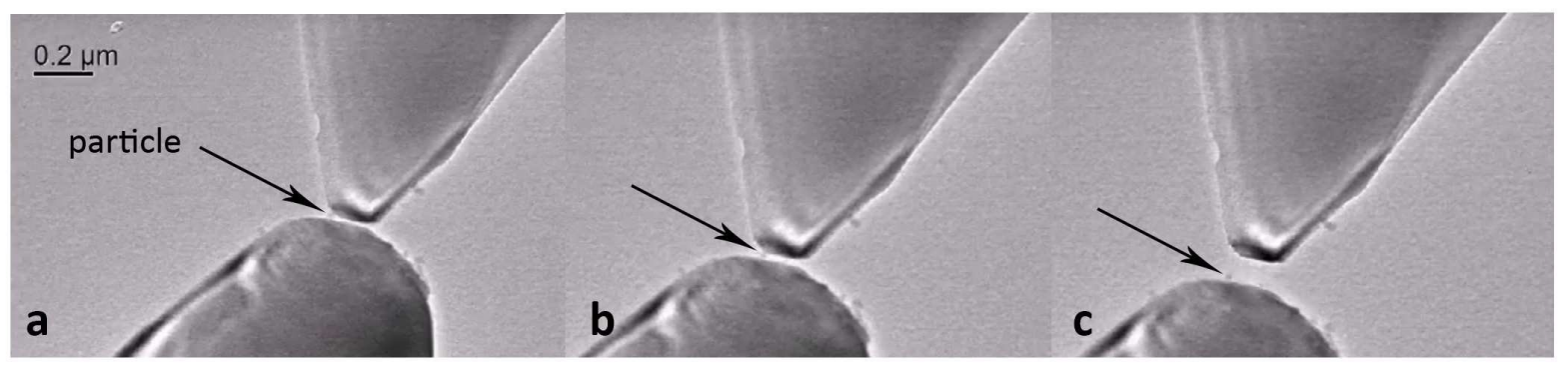

Figure S4: Frames from a contact-separate test from the third group which had a particle in contact that was transferred from the upper surface to the lower surface with negligible adhesion. a Making contact with the particle sandwiched between the surfaces. b Pulling the upper and lower probes apart. c Several frames after contact separation. The particle is now on the lower probe. This test is between 41:47 and 41:51 in the video. Adhesion from this contact-separate test was below the detectable limit. constant $[1,2]$. The uncertainty in $d_{\text {pull-off }}$ is the sum of the uncertainties from the finite frame rate, finite pixel size, and from the image-correlation code i.e., $\Delta d_{p u l l-o f f}=$ $\Delta d_{\text {frame-rate }}+\Delta d_{\text {pixel-size }}+\Delta d_{\text {code }}$.

The frame rate uncertainty $\Delta d_{\text {frame-rate }}$ is no greater than the product the maximum retraction speed, $13 \frac{\mathrm{nm}}{\mathrm{s}}$, and the inverse of the frame rate, $\frac{1}{2.9} \frac{\mathrm{s}}{\text { frame }}$ i.e., $\frac{13}{2.9} \frac{\mathrm{nm}}{\text { frame }}=4.48 \frac{\mathrm{nm}}{\text { frame }}$. This means there was at most $4.48 \mathrm{~nm}$ of travel in between the frame before pull-off apparently started and the frame at which it apparently started, so $\Delta d_{\text {frame-rate }}$ $=4.48 \mathrm{~nm}$. The pixel size uncertainty is no greater than the distance per pixel at the lowest magnification used in all experiments, 50,000x. For our JEOL 2010F TEM, this is $0.27 \frac{\mathrm{nm}}{\text { pixel }}$. Since there is at most one pixel of uncertainty, $\Delta d_{\text {pixel-size }}=0.27 \mathrm{~nm}$. This is comparable to the point-to-point resolution of the JEOL $2010 \mathrm{~F}$ of $0.19 \mathrm{~nm}$. The uncertainty in the pull-off distance from the image-correlation code is no greater than $1 \mathrm{~nm}$.

The largest source of uncertainty comes from the cantilever calibration. Per Refs. [1,2], the uncertainty is within ten percent of the nominal spring constant i.e., $\Delta k_{n}=0.1 \times k_{n}$. Since $d_{\text {pull-off }}=\frac{F_{\text {pull-off }}}{k_{n}}$, the second term in

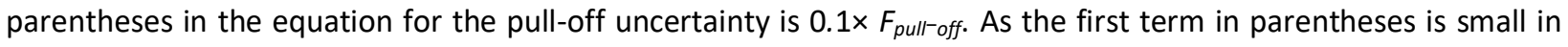
comparison to the second term for all but the smallest pull-off forces, it can be generally said that the uncertainty of the pull-off force stems mostly from the uncertainty in the calibrated spring constant of the cantilever, so the uncertainty is $10 \%$ of the pull-off force. Figure S5 is a plot of the uncertainty of the pull-off force against the pull-off force, illustrating that $\Delta F_{\text {pull-off }} \approx 0.1 \times F_{\text {pull-off }}$ 
Though an upper limit of $5.2 \mathrm{nN}$ for the pull-off force from tests where adhesion was nominally zero was estimated, the actual uncertainty in pull-off force from such tests is probably much less than this upper bound as there is no indication, such as increased blurriness in the upper probe, that it adhered strongly enough to the lower probe to be retracted slightly as the lower probe was actuated away from it.

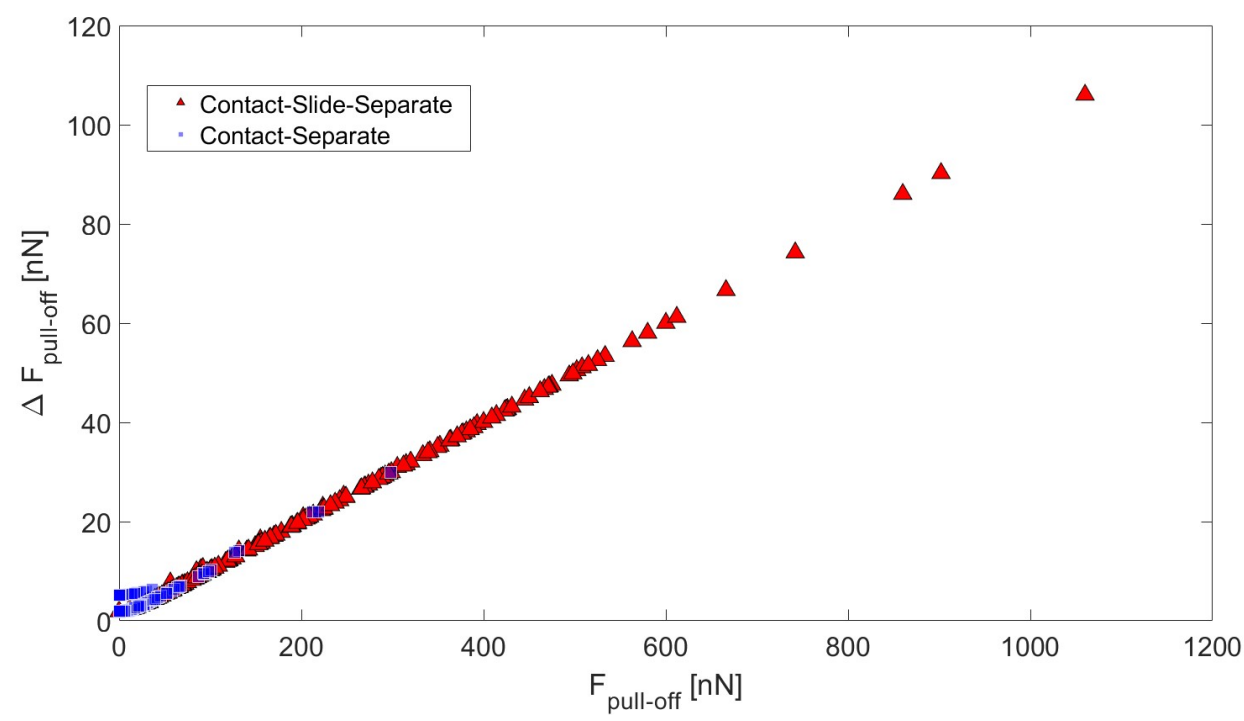

Figure S5: The uncertainty in the pull-off force, vs. the pull-off force, demarcated by whether sliding was induced.

\section{Bibliography}

[1] J. E. Sader, I. Larson, P. Mulvaney, and L. R. White, "Method for the calibration of atomic force microscope cantilevers," Review of Scientific Instruments, vol. 66, no. 7, pp. 3789-3798, 1995.

[2] T. D. B. Jacobs and R. W. Carpick, "Nanoscale wear as a stress-assisted chemical reaction.," Nature Nanotechnology, vol. 8, pp. 108-12, Feb. 2013. 